Article

\title{
Analysis of the PAPR Behavior of the OFDM Passband Signal
}

\author{
Frank Andrés Eras 0000-0002-7844-5670, Italo Alexander Carreño, Thomas Borja, Diego Javier \\ Reinoso* 0000-0003-0854-1250, Luis Felipe Urquiza 0000-0002-6405-2067, Martha Cecilia \\ Paredes 0000-0001-5789-4568 \\ Departamento de Electrónica, Telecomunicaciones y Redes de Información, Escuela Politécnica Nacional \\ * Correspondence: diego.reinoso@epn.edu.ec
}

\begin{abstract}
Orthogonal Frequency Division Multiplexing (OFDM) is a technique widely used in today's wireless communication systems due to its ability to combat the effects of multi-path in the signal. However, one of the main limitations of the use of OFDM is its high Peak-to-Average Power Ratio (PAPR), which reduces the efficiency of the OFDM system. The effects of PAPR can produce both out-of-band and in-band radiation, which degrades the signal by increasing the bit error rate (BER), this occurs in both baseband and bandpass sginals. In this document the effect of the PAPR in a OFDM passband signal is analyzed considering the implementation of a High Power Amplifier (HPA) and the Simple Amplitude Predistortion-Orthogonal Pilot Sequences (OPS-SAP) scheme to reduce the PAPR.
\end{abstract}

Keywords: OFDM; PAPR; passband; IEEE 802.11p

\section{Introduction}

For some years, many wireless communication systems have based the transmission of information on the Orthogonal Frequency Division Multiplexing (OFDM) scheme, which is used in standards such as Long-Term Evolution (LTE), Wireless Local Area Networks (WLAN), Digital Video Broadcasting (DVB-T), among others. The use of OFDM is due to the multiple advantages it offers against the effects of the wireless environment, however, one of the problems of OFDM is the well-known high Peak-to-Average Power Ratio (PAPR) that degrades the signal at the time of passing through a non-linear High Power Amplifier (HPA) [1].

Over the years, different techniques have been developed that have allowed the reduction of PAPR, being possible to apply these techniques to the passband signal, which carries the information on a much higher frequency. Being of interest the changes that the PAPR has, specifically comparing baseband with passband, before and after the application of the PAPR reduction technique.

For this work, the Simple Amplitude Predistortion-Orthogonal Pilot Sequences (OPS-SAP) technique was selected and implemented, this technique consists of the OPS distortion-free technique in combination with the SAP algorithm [2].

The rest of this document is organized as follows. Section 2 provides a theoretical review of the main elements for the understanding of the work, such as OFDM, PAPR and HPA. Section 3 explains how the different parts were implemented in MATLAB. Section 4 discusses the results of the simulations performed. Finally, in Section 5 the corresponding conclusions can be observed. 


\section{Theoretical Review}

\subsection{The PAPR problem of an OFDM signal}

OFDM is a multi-carrier technique that is used in various wireless communications scenarios because it offers great advantages in the transmission of information, such as robustness against the effects of multipath, high spectral efficiency and its simple equalizer structure [3]. However, as it has advantages, OFDM has some drawbacks, such as: Symbol Time Offset (STO) for time synchronization accuracy and Carrier Frequency Offset (CFO) for frequency synchronization accuracy, which are necessary to maintain orthogonality between subcarriers [4].

The greatest limitation when transmitting with an OFDM system occurs when there are too high-power peaks with respect to the average power in the transmission, which leads to large fluctuations in the OFDM signal. These peaks are formed when subcarriers with the same phase add up in a certain time and therefore there is degradation in the signal, especially when the signal passes through a non-linear amplifier for example HPA [5]. This limitation is known as Peak-to-average power ratio (PAPR). In general, the PAPR, denoted as $X$ in the time domain is mathematically defined as the existing relationship between the maximum instantaneous power and its average power [6], which can be described with the following expression:

$$
\chi=\operatorname{PAPR}\{x(t)\}=\frac{\max _{0 \leq t \leq T}|x(t)|^{2}}{E\left\{|x(t)|^{2}\right\}},
$$

\footnotetext{
Where $\max _{0 \leq t \leq T}|x(t)|^{2}$ is the maximum instantaneous power, $E\left\{|x(t)|^{2}\right\}$ is the average signal power and $E\{\cdot\}$ denotes the expected value[5].
}

In real OFDM systems, usually the discrete time model is implemented, therefore, is more convenient to work with the PAPR in discrete time. So, the PAPR is mathematically defined as [7]:

$$
\chi=\operatorname{PAPR}\{x[n]\}=\frac{\max _{0 \leq n \leq N-1}|x[n]|^{2}}{E\left\{|x[n]|^{2}\right\}} .
$$

\subsection{PAPR in passband signal}

A OFDM passband signal is usually transmitted with a frequency much higher than the bandwidth of each subcarrier of the baseband signal, $f_{c} \gg \Delta f$, where $f_{c}$ is the carrier frequency, therefore, the maximum of the passband signal is approximately equal to the maximum of the baseband signal in continuous time [6] mathematically defined as:

$$
\max \left|x_{\mathrm{PB}}(t)\right| \approx \max |x(t)|
$$

As in the base band, in passband it is possible to obtain the average power, which is given by:

$$
\begin{aligned}
E\left\{\left|x_{\mathrm{PB}}(t)\right|^{2}\right\} & =E\left\{\left|\Re\left\{x(t)^{j 2 \pi f_{c} t}\right\}\right|^{2}\right\} \\
& \left.=\left.E\left\{\mid x_{R}(t) \cos \left(2 \pi f_{c} t\right)-x_{I}(t) \sin \left(2 \pi f_{c} t\right)\right\}\right|^{2}\right\} \\
& =\frac{1}{2} E\left\{|x(t)|^{2}\right\} .
\end{aligned}
$$

Analyzing the above expressions, it can be affirmed that the PAPR of the complex passband signal $\chi_{\mathrm{PB}}$ can be approximate to [7]:

$$
\chi_{\mathrm{PB}} \approx 2 \chi
$$




\subsection{High Power Amplifier}

In many radio systems today, HPAs are used on the transmitter side to achieve enough transmission power to reach the established parameters. HPAs usually operate near the saturation point to obtain maximum performance, however, they are devices limited in power and quite sensitive to the variation in signal amplitude[7].

If the system works with an OFDM signal with large fluctuations in its envelope, this will cause the HPA to saturate, causing radiation, both outside and inside the band, which affects adjacent bands and the signal itself. In order to avoid these problems, the HPA must work below its saturation point (back-off), which reduces the efficiency of this device.

The HPA being a physical device will introduce distortion in the OFDM signal, to measure this distortion the terms Input Back-off (IBO) and (Output Back-off (OBO) are defined, which are mathematically defined as [8]:

$$
\begin{gathered}
\mathrm{IBO}=10 \log _{10} \frac{P_{\text {in }}^{\max }}{P_{\text {in }}}[\mathrm{dB}] \\
\mathrm{OBO}=10 \log _{10} \frac{P_{\text {out }}^{\max }}{P_{\text {out }}}[\mathrm{dB}]
\end{gathered}
$$

Where $P_{\text {in }}^{\max }$ and $P_{\text {out }}^{\max }$ represent the maximum instantaneous power input and output, respectively, and $P_{\text {in }}$ and $P_{\text {out }}$ represent the average input and output power, respectively, of the HPA [7].

For the present work the Rapp model was used, which simulates a Solid-State Power Amplifier (SSPA) that produces a smooth transition from the envelope modulation to the saturation level[5].

\subsection{Complementary Cumulative Distribution Function (CCDF)}

The Complementary Cumulative Distribution Function (CCDF) is quite used today to evaluate PAPR reduction techniques. The CCDF determines the probability that the PAPR exceeds a given value or threshold $X_{0}$. The CCDF can be written as [9]:

$$
\operatorname{CCDF}\{\chi\}=\operatorname{Pr}\left\{\chi \geq \chi_{0}\right\}=1-\left(1-e^{-\chi_{0}^{2}}\right)^{N}
$$

\subsection{PAPR reduction techniques}

Currently, several techniques have been proposed to reduce PAPR in OFDM systems, being widely classified into techniques that introduce signal distortion, and those that do not introduce signal distortion. Each of the techniques has its advantages and disadvantages, however, its analysis is not covered in this work.

One of the main techniques to reduce PAPR is the OPS-SAP, with which it is possible to move certain constellation points of the OFDM symbol to counteract the PAPR[5].

\subsubsection{OPS-SAP technique}

This technique for reducing PAPR is based on a two-step algorithm, where the OPS is implemented in the first step and then SAP is added as the second step. In the first stage the sequence of pilots that offers the lowest PAPR, of the whole set of available pilots, is inserted. In the second step, the extension of certain symbols in the frequency domain is performed, the symbols to be extended are chosen by means of a metric, which measures the contribution of the frequency symbols that have large power peaks in the time domain [2].

\section{Matlab}

To perform the analysis, several scripts were implemented in MATLAB that simulate the IEEE 802.11p [10] physical layer and the PAPR reduction techniques described above, also a script to obtain 
the CCDF was implemented in order to evaluate the PAPR reduction technique. Figure 1 shows the process that the data must follow for its transmission, this process includes a scrambler, a convolutional coder, a interleaver, a modulator, the generation of OFDM symbols, the respective IFFT, the PAPR reduction technique block, the cyclic prefix aggregation, frequency change to passband and the HPA. All these blocks follow the indications of the IEEE 802.11p standard [10].

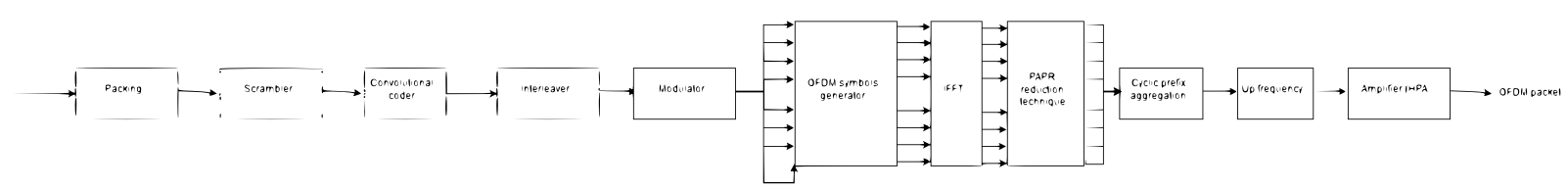

Figure 1. Transmittion Block Diagram

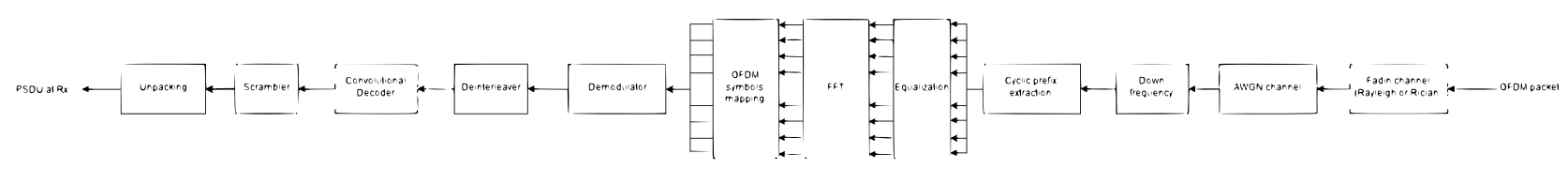

Figure 2. Reception Block Diagram

\section{Simulation Results}

Figures 3, 4, 5 and 6 present PER vs SNR curves with different data rates. Dashed line curves represent amplified OFDM frames without the application of PAPR reduction technique whilst solid line curves represent amplified OFDM frames with the application of PAPR reduction technique. These graphs show a slight change in PER performance. The most noticeable change is observed on Figure 5 which refers to PER vs SNR for passband OFDM frames with a carrier frequency of $5.9 \mathrm{GHz}$. The PER is slightly improved for frames with QPSK and 3/4 code rate and 16QAM with 3/4 code rate. Moreover, passband frames with 64QAM modulation present distortion (Figures 5 and 7) in its PER which leads to the conclusion that applying a HPA amplifier causes this result.

\section{CCDF curves analysis}

The PAPR reduction technique called OPS-SAP was applied on these OFDM frames which consists of the creation of a finite number of orthogonal pilot sequences [1]. For each OFDM symbol a pilot sequence is selected to get the lowest PAPR when combined with the data in the modulation process. To check its effect, a comparison between the CCDF curves from frames with and without technique is carried out. 


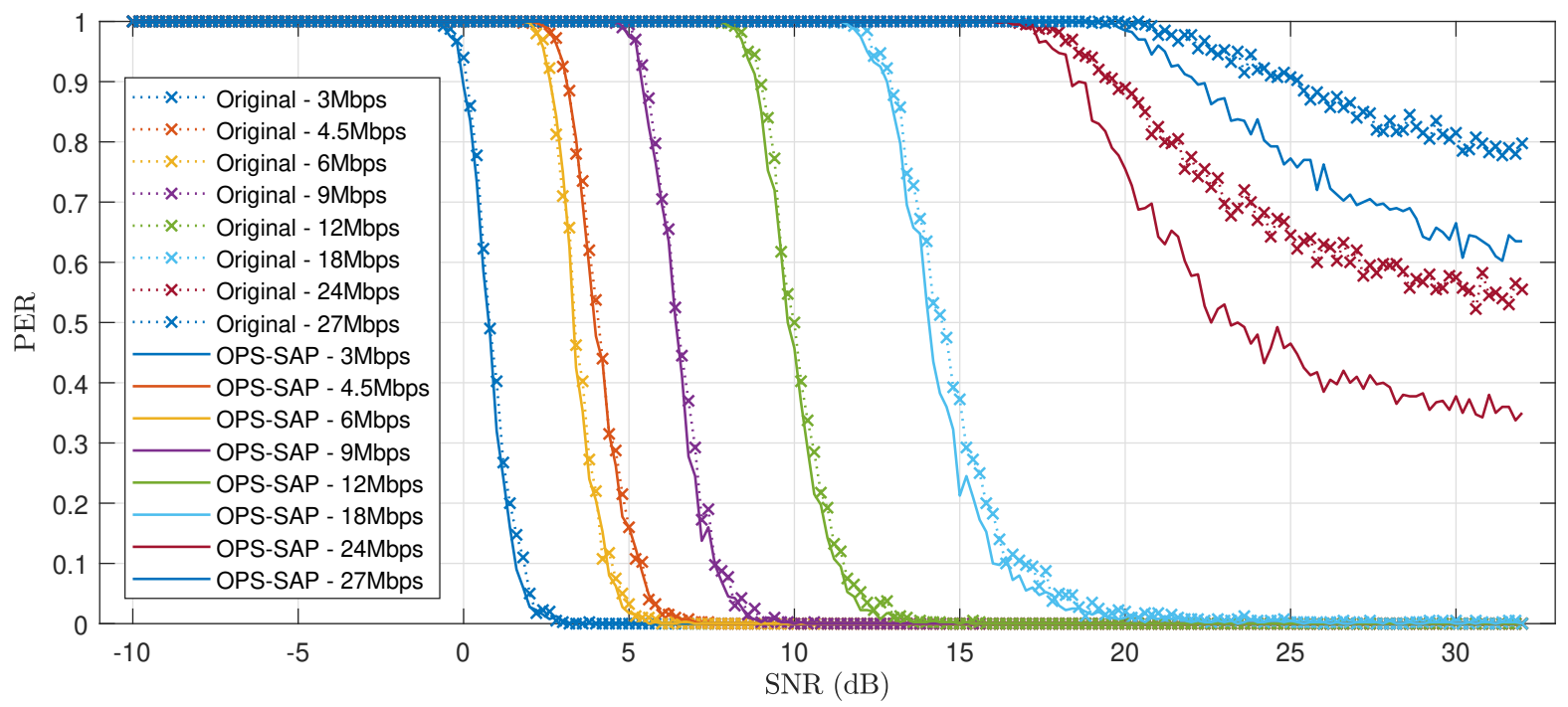

Figure 3. PER vs SNR of an amplified passband signal $(2.4 \mathrm{GHz})$.

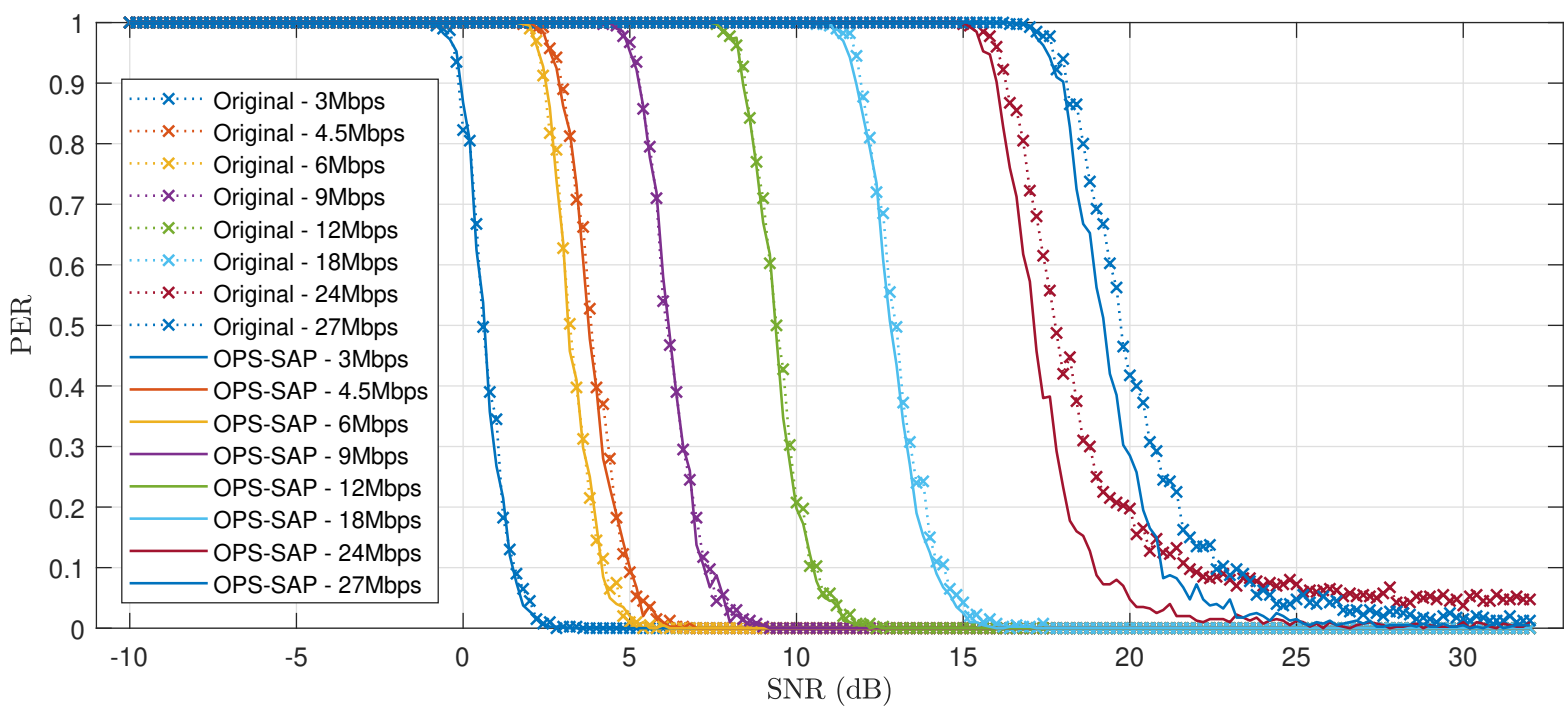

Figure 4. PER vs SNR of an amplified baseband signal (2.4GHz). 


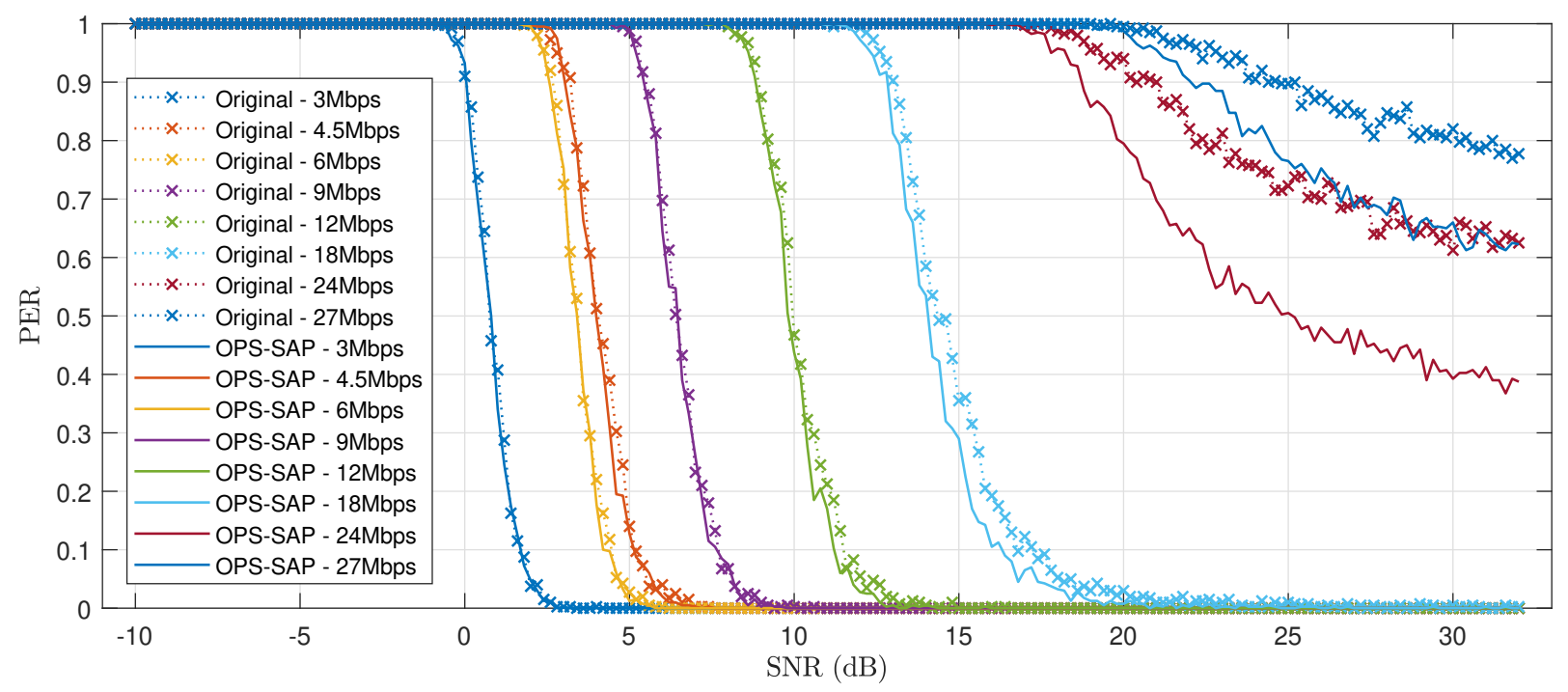

Figure 5. PER vs SNR of an amplified passband signal (5.9GHz)..

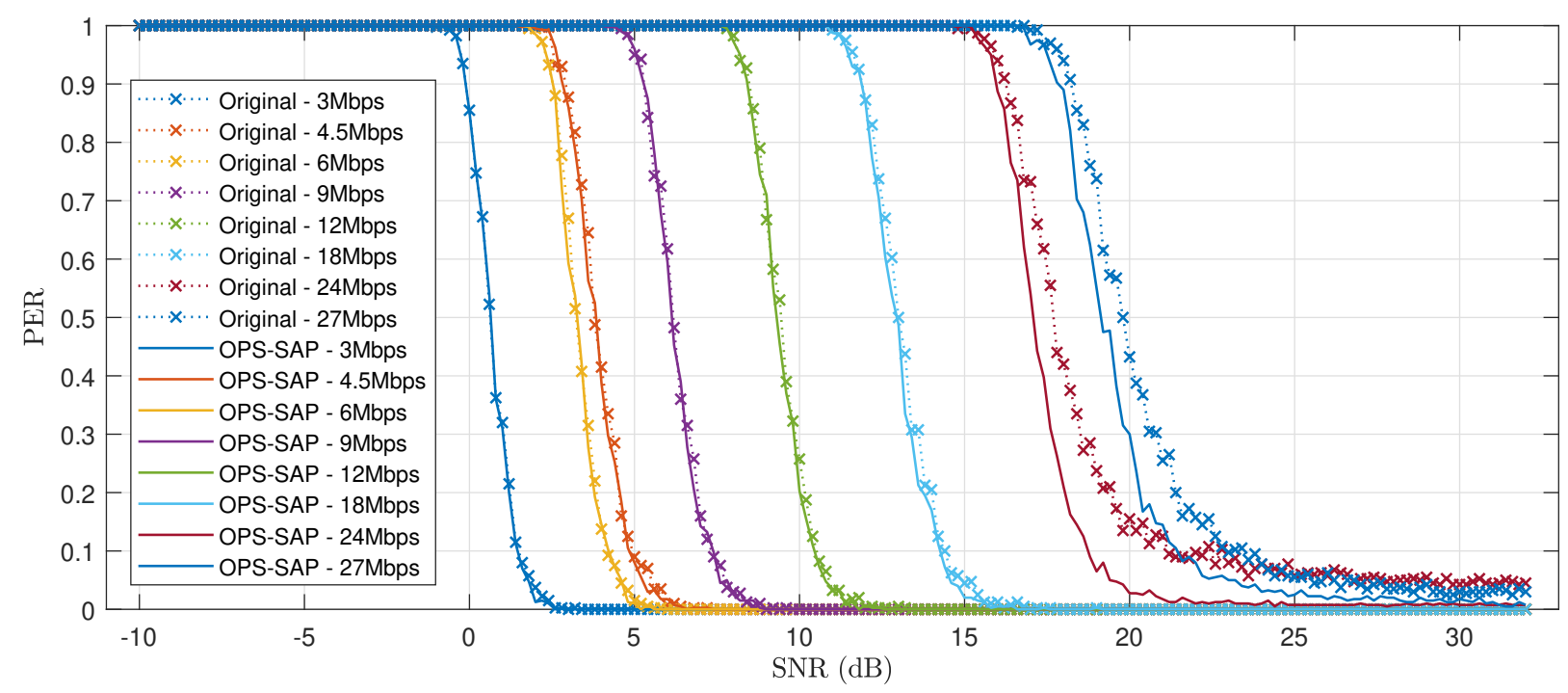

Figure 6. PER vs SNR of an amplified baseband signal (5.9GHz case).

Figures 7 and 8 show the CCDF curves of baseband OFDM frames at different rates. Dashed line curves represent OFDM frames without the application of PAPR reduction technique whilst solid line curves represent OFDM frames with the application of PAPR reduction technique. For a probability of $10^{-2}$ it is observed a reduction of $0.6 \mathrm{~dB}$ for OFDM frames at $3 \mathrm{Mbps}, 0.4 \mathrm{~dB}$ for OFDM frames at $4.5 \mathrm{Mbps}, 0.8 \mathrm{~dB}$ for OFDM frames at $6 \mathrm{Mbps}, 1.2 \mathrm{~dB}$ for OFDM frames at $9 \mathrm{Mbps}, 0.4 \mathrm{~dB}$ for OFDM frames at $12 \mathrm{Mbps}, 0.3 \mathrm{~dB}$ for OFDM frames at $18 \mathrm{Mbps}, 1.15 \mathrm{~dB}$ for OFDM frames at $24 \mathrm{Mbps}$ and a reduction of $0.3 \mathrm{~dB}$ for OFDM frames at $27 \mathrm{Mbps}$. OFDM frames with QPSK and a code rate of 3/4 present the best performance. 


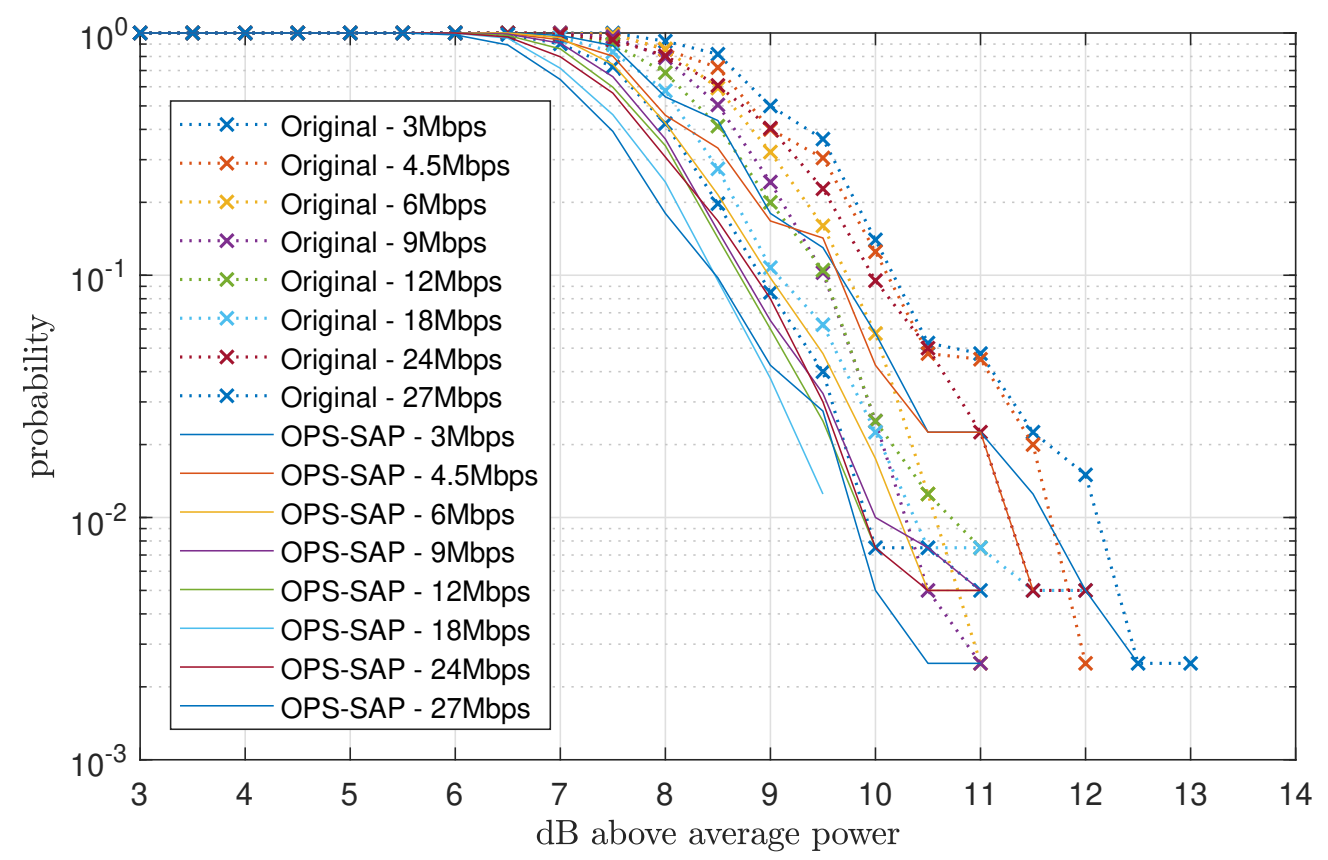

Figure 7. CCDF curve of baseband OFDM frames (2.4GHz case).

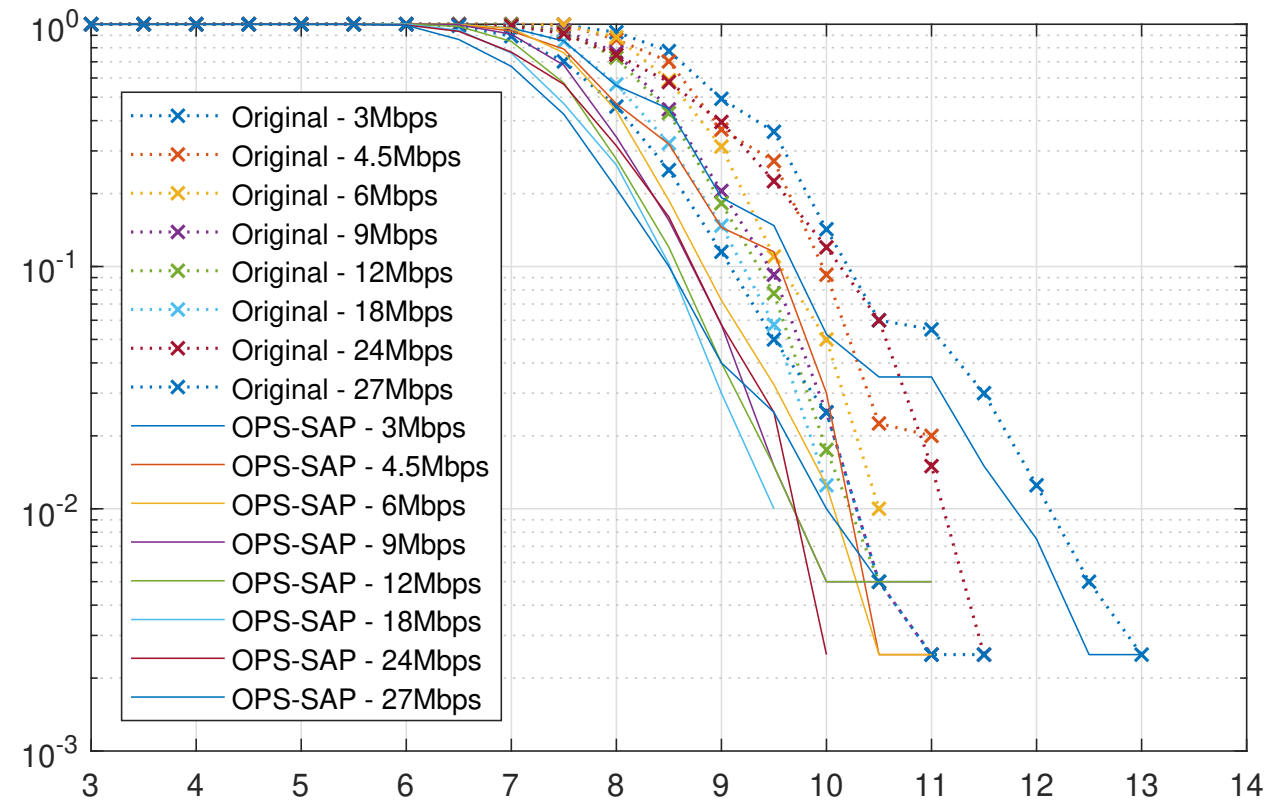

Figure 8. CCDF curve of baseband OFDM frames (5.9GHz case).

Figures 9 and 10 show the CCDF curves of passband OFDM frames with a carrier frequency of $2.4 \mathrm{GHz}$ and $5.9 \mathrm{GHz}$, respectively at different rates. For the $2.4 \mathrm{GHz}$ case and for a probability of $10^{-2}$ it is observed a reduction of $0.8 \mathrm{~dB}$ for OFDM frames at $3 \mathrm{Mbps}, 0.8 \mathrm{~dB}$ for OFDM frames at $4.5 \mathrm{Mbps}$, $0.9 \mathrm{~dB}$ for OFDM frames at $6 \mathrm{Mbps}, 0.9 \mathrm{~dB}$ for OFDM frames at $9 \mathrm{Mbps}, 0.7 \mathrm{~dB}$ for OFDM frames at 12 Mbps, $0.2 \mathrm{~dB}$ for OFDM frames at $18 \mathrm{Mbps}, 1.4 \mathrm{~dB}$ for OFDM frames at $24 \mathrm{Mbps}$ and a reduction of 0.8 $\mathrm{dB}$ for OFDM frames at $27 \mathrm{Mbps}$. Here, OFDM frames with 64QAM and a code rate of $2 / 3$ present the best performance.

For the $5.9 \mathrm{GHz}$ case and for a probability of $10^{-2}$, it is observed a reduction of $0.6 \mathrm{~dB}$ for OFDM 
frames at $3 \mathrm{Mbps}, 0.5 \mathrm{~dB}$ for OFDM frames at 4.5 Mbps, $0.6 \mathrm{~dB}$ for OFDM frames at $6 \mathrm{Mbps}, 0.7 \mathrm{~dB}$ for OFDM frames at $9 \mathrm{Mbps}, 0.7 \mathrm{~dB}$ for OFDM frames at $12 \mathrm{Mbps}, 0.9 \mathrm{~dB}$ for OFDM frames at $18 \mathrm{Mbps}$, $0.02 \mathrm{~dB}$ for OFDM frames at $24 \mathrm{Mbps}$ and a reduction of $0.3 \mathrm{~dB}$ for OFDM frames at $27 \mathrm{Mbps}$. OFDM frames with 16QAM and a code rate $3 / 4$ present the best performance.

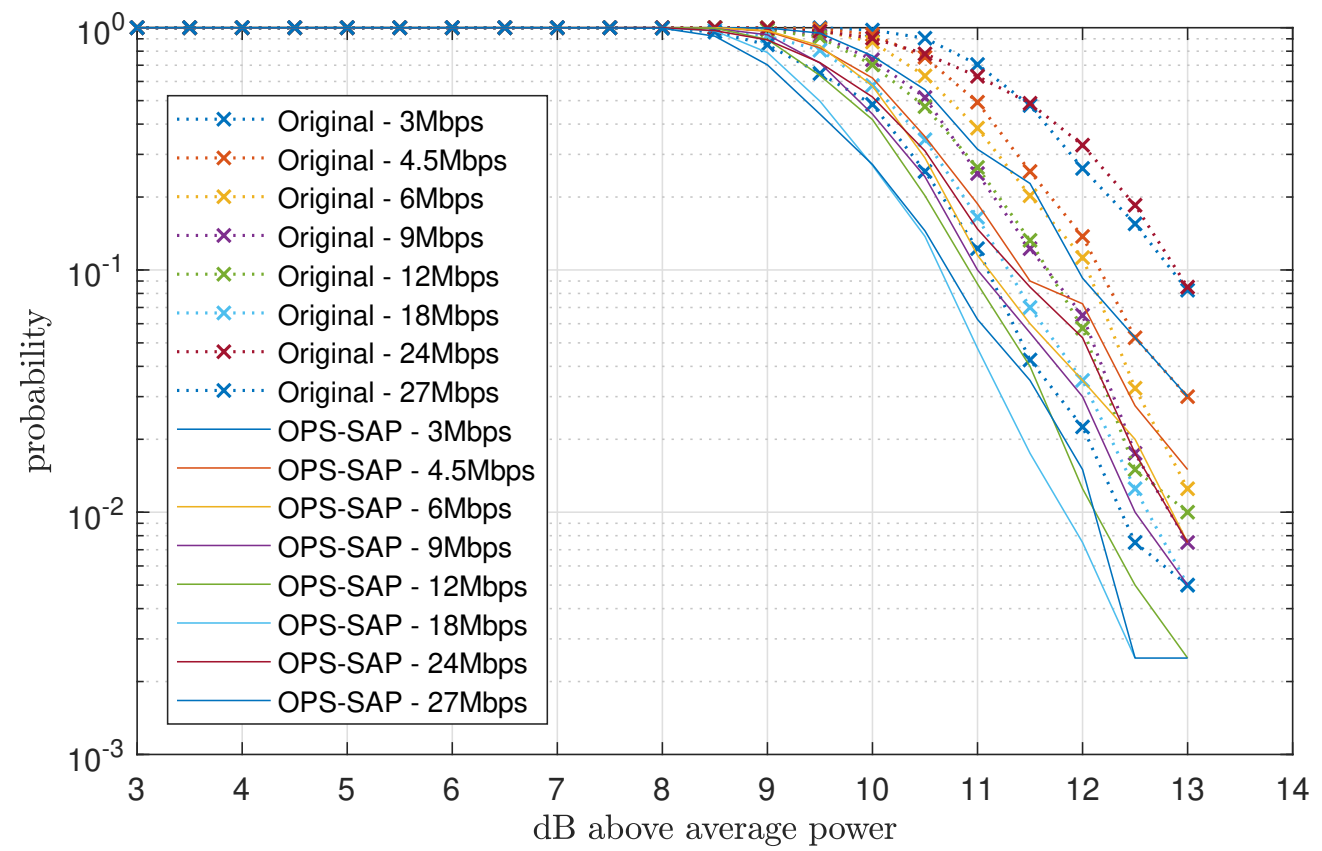

Figure 9. CCDF curve of passband OFDM frames (2.4GHz case).

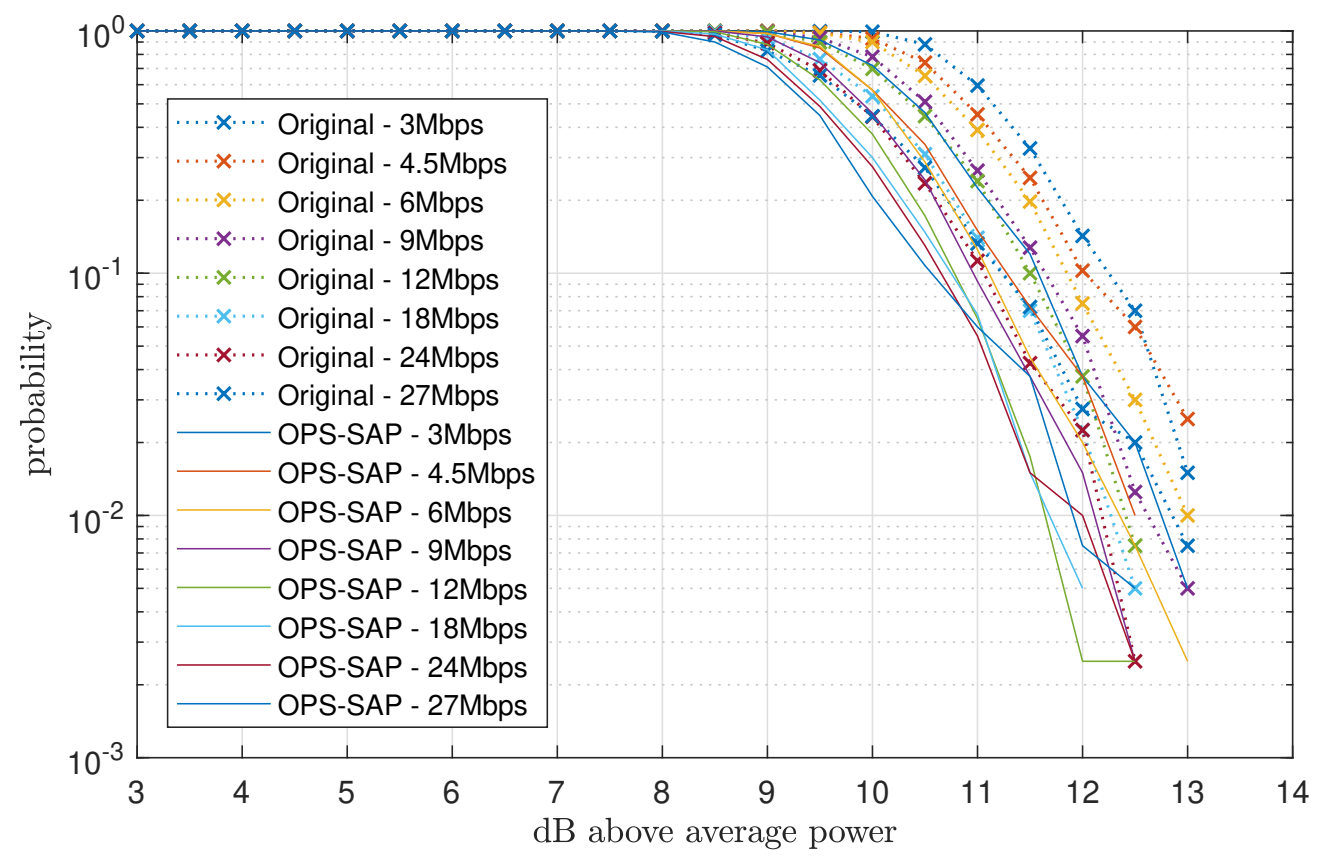

Figure 10. CCDF curve of passband OFDM frames (5.9GHz case).

\section{Conclusions}

- In this work, the behavior of the PAPR in passband signal is evaluated in an environment that uses OFDM with the IEEE 802.11p physical layer. The OPS-SAP technique is applied for PAPR 
reduction and it presents a slight better performance working with passband than with baseband for certain modulation schemes and coding rates.

- Applying the PAPR reduction technique in bandpass signals has a similar result to a baseband signal as mentioned earlier, except for high speeds such as $24 \mathrm{Mbps}$ and $27 \mathrm{Mbps}$, in which there is a considerable distortion in the passband signal, due to the HPA amplifier used.

Acknowledgments: The authors gratefully acknowledge the financial support provided by the Escuela Politécnica Nacional, for the development of the project PIJ-16-01 - "Modelamiento de la Packet Error Rate (PER) incluyendo condiciones de Peak-to-Average Power Ratio (PAPR) para transmisiones Ad-Hoc".

\section{References}

1. Paredes Paredes, M.C.; Escudero-Garzás, J.J.; Fernández-Getino García, M.J. PAPR Reduction via Constellation Extension in OFDM Systems Using Generalized Benders Decomposition and Branch-and-Bound Techniques. IEEE Transactions on Vehicular Technology 2016, 65, 5133-5145. doi:10.1109/TVT.2015.2450178.

2. Paredes Paredes, M.C.; Fernandez-Getino Garcia, M.J. Energy efficient peak power reduction in OFDM with amplitude predistortion aided by orthogonal pilots. IEEE Transactions on Consumer Electronics 2013, 59, 45-53. doi:10.1109/TCE.2013.6490240.

3. Asif, S. Next Generation Mobile Communications Ecosystem: Technology Management for Mobile Communications; 2011. doi:10.1002/9780470972182.

4. Yong Soo Cho, Jaekwon Kim, W.Y.Y.C.G.K. MIMO-OFDM Wireless Communications with MATLAB®; 2010; p. 544.

5. Paredes, M.C.P.; Grijalva, F.; Carvajal-Rodríguez, J.; Sarzosa, F. Performance analysis of the effects caused by HPA models on an OFDM signal with high PAPR. 2017 IEEE Second Ecuador Technical Chapters Meeting (ETCM), 2017, pp. 1-5. doi:10.1109/ETCM.2017.8247511.

6. Tellado, J. Multicarrier modulation with low PAR: applications to DSL and wireless; Kluwer Academic Publishers Norwell, MA, USA (C)2000, 2000; p. 152.

7. Paredes, M.C.P. Algoritmos para la reducción de los picos de potencia en los sistemas OFDM. PhD thesis, Universidad Carlos III de Madrid, Escuela Politécnica Superior, Departamento Teoría de la señal y comunicaciones, 2014.

8. Litsyn, S. Peak Power Control in Multicarrier Communications; Cambridge University Press, 2007. doi:10.1017/CBO9780511618383.

9. Ochiai, H.; Imai, H. On the distribution of the peak-to-average power ratio in OFDM signals. IEEE Transactions on Communications 2001, 49, 282-289. doi:10.1109/26.905885.

10. IEEE Standard for Information technology-Telecommunications and information exchange between systems Local and metropolitan area networks-Specific requirements Part 11: Wireless LAN Medium Access Control (MAC) and Physical Layer (PHY) Specifications. IEEE Std 802.11-2012 (Revision of IEEE Std 802.11-2007) 2012, pp. 1-2793. doi:10.1109/IEEESTD.2012.6178212. 